\title{
Factores que inciden en el proceso de aprendizaje del Inglés como lengua extranjera
}

\section{Factors that affect the process of learning from English as a foreign language}

\author{
MSc, Jorge Humberto Tapia Celi ${ }^{1}$ \\ jorge.tapiac@ug.edu.ec
}

Recibido: 1/12/2017, Aceptado: 1/02/2018

\begin{abstract}
RESUMEN
Este artículo hace una alusión concisa, pero también con algunos de los detalles que tuvieron que ver con el trabajo de investigación. Trabajo este, que tiene como objetivos principales dos: el primero convertirse en una Tesis Doctoral y el segundo servir de guía conductora a las directivas y a los profesores de la Facultad de Filosofía, Letras y Ciencias de la Educación de la Universidad de Guayaquil, quienes serán los encargados de diseñar los programas de enseñanza/aprendizaje de inglés, como segunda lengua, y desarrollarlos. Algunos de los temas acá tratados fueron los más importantes del trabajo de investigación, en sí mismo, son el de la Validación de Expertos sobre un instrumento de recogida de datos, la Revisión de la Literatura que sirvió, entre otras cosas, para determinar el imprescindible Marco Teórico, y los Resultados de las encuestas, por supuesto.
\end{abstract}

Palabras clave: trabajo de investigación, segunda lengua, programa de enseñanza/aprendizaje

\begin{abstract}
This paper makes a concise reference, but also with some of the details that had to do with research work. This work, which has two main objectives: the first to become a Doctoral thesis and the second serve as conductive policies and guide the teachers of the Faculty of Philosophy, Arts and Sciences of Education of the University of Guayaquil, who will be responsible for designing programs of teaching and learning of English as a Second Language, and developing them. Some of the topics discussed here were the most important in the research work, in itself, they are the experts of validation on an instrument for collection of data, the review of literature which served, among other things, to determine the essential theoretical framework, and the results of the polls, of course.
\end{abstract}

Keywords: research work, second language, teaching and learning program

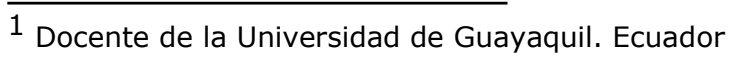




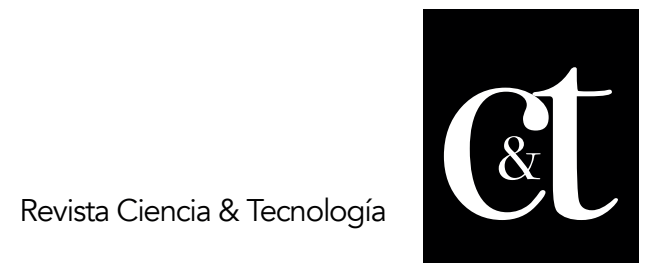

No. 18,30 de abril de 2018

ISSN impreso: 1390 - 6321

\section{Introducción}

No es suficiente que un alumno tenga talento en las áreas lógico-matemática, lingüística o en lenguas extranjeras, sino que la sociedad reclama una nueva forma de pensamiento, una formación basada en el conjunto de materias del currículo tanto las que se potencian en el hemisferio cerebral izquierdo como en el derecho para formar a personas que sepan desenvolverse en diversas situaciones, entre diferentes colectivos, que sean autónomas y talentosas en lo que les apasione. Este tipo de inteligencias se desarrollan dependiendo de tres factores (Armstrong, 1999) por la dotación biológica, que es en la que se incluyen los factores genéticos o hereditarios y los daños o heridas que el cerebro haya podido sufrir durante o después del nacimiento; por otro lado, la historia de la vida personal donde se incluyen experiencias que el niño haya podido tener tanto con los padres, con los profesores, con el resto de compañeros u otras personas que le hayan dado la oportunidad de desarrollar o reducir su potencial o talento; y por último, el antecedente cultural e histórico, que se incluye la época y el lugar donde nació y se crió (Paíno, 2017, pág. 16).

Un método de validación útil para verificar la fiabilidad de una investigación que se define como "una opinión informada de personas con trayectoria en el tema, que son reconocidas por otros como expertos cualificados en éste, y que pueden dar información, evidencia, juicios y valoraciones" (Escobar-Pérez y Cuervo-Martínez, 2008:29). Tras someter un instrumento de cotejo a la consulta y al juicio de expertos éste ha de reunir dos criterios de calidad: validez y fiabilidad. La validez de contenido se establece con frecuencia a partir de dos situaciones, una que atañe al diseño de una prueba y, la otra, a la validación de un instrumento sometido a procedimientos de traducción y estandarización para adaptarlo a significados culturales diferentes. Es aquí donde la tarea del experto se convierte en una labor fundamental para eliminar aspectos irrelevantes, incorporar los que son imprescindibles y/o modificar aquellos que lo requieran (Robles y Rojas, 2015, pág. única).

La Validación de Expertos, a la cual se acude, hasta el punto de colocarlo al inicio del presente acápite, por un motivo de que este trabajo de investigación se convierta en una Tesis Doctoral, es evidente que se pretende que la Facultad implemente los cursos de inglés propuestos. Y se pretende, de un lado, porque es una necesidad sentida y, de otra parte, porque, una vez implementados por parte de la Facultad, el ejemplo será seguido por otras Facultades de la Universidad de Guayaquil. De esta manera, entonces, se tendrá una universidad inmersa, como se merece por su enorme trayectoria, en el siglo XXI, en cuanto a que tendrá, como estudiantes y como futuros profesionales graduados, a personas que se pueden contactar con el mundo de forma clara, fluida y sin obstáculos innecesarios.

De los cuatro factores examinados mediante la encuesta la Motivación es el más importante de todos los factores analizado, cuando se trata de los estudiantes de la Facultad de Filosofía, Letras y Ciencias de la Educación en mención, es más que evidente que existe un grado muy importante de motivación para aprender inglés. La causa de este último fenómeno es una circunstancia con insistencia en esta Ponencia y, también, durante casi todos los capítulos del trabajo de investigación mismo.

Se trata de que los estudiantes de la Facultad son conscientes, incluso mucho más que el alumnado de otras Facultades de la Universidad de Guayaquil, de la imperiosa necesidad de estudiar inglés. 


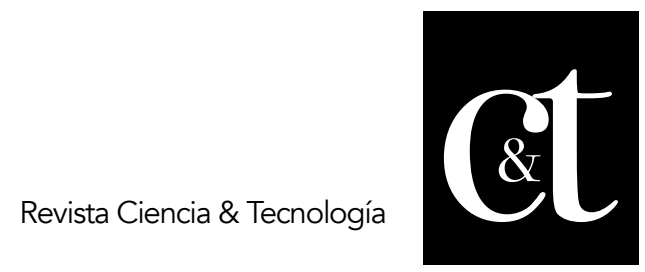

No. 18, 30 de abril de 2018

ISSN impreso: 1390 - 6321

Quienes deseen perfeccionar su nivel actual de inglés $y$, de paso, enfocarse en un nivel relacionado con la Filosofía y la Literatura, lo podrán conseguir al adherirse a los cursos. Ello, por cuanto, como es de esperar, las directivas y el personal de docentes diseñarán dichos cursos, teniendo en consideración, precisamente, que se trata de estudiantes de estas disciplinas, más que de las mismas Ciencias de la Educación. No se recomienda, al momento de proceder con el diseño de los cursos, las directivas y los profesores se desentiendan de un elemento: que, según los resultados obtenidos luego de efectuar las encuestas, se percibe un alto grado de heterogeneidad en lo que respecta del nivel de inglés, entre el estudiantado. Hay dos factores trascendentales: de un lado, la pre-aludida heterogeneidad $y$, de otra parte, la necesidad de enfocar los cursos hacia dos disciplinas, esto es, hacia la Filosofía y la Literatura.

¿Será demasiado compleja la tarea de enseñar un inglés enfocado a dos disciplinas de semejante envergadura, pero con alumnos que están bien disparejos, entre sí, en lo que respecta a los conocimientos actuales del más universal de los idiomas? Sí puede ser, pero no es algo insuperable. Tal y como se ha manifestado, a lo largo de muchos apartes del presente trabajo de investigación, con pretensión de convertirse en la Tesis Doctoral, la Motivación es el mejor "ingrediente" posible para superar barreas planteadas. Sin embargo, es imprescindible que la motivación misma esté acompañada, muy de cerca, con ciertas estrategias pedagógicas. Así, por ejemplo, enseñar inglés haciendo uso de la literatura, resulta de lo más agradable para los estudiantes y para los profesores. En resumidas cuentas: sí existen métodos y estrategias para incrementar la motivación como consecuencia lógica, intentar "igualar" lo que, se encuentra de hecho desigual.

La edad: Se argumenta sobre el tema de la heterogeneidad de la edad de los alumnos de la Facultad, es que, en general, las investigaciones dirigidas al estudio de los factores que inciden en el rendimiento académico de los estudiantes universitarios incorporan una amplia variedad de posibles determinantes. En este estudio se consideran desde los factores de carácter socio-demográfico (género, edad, formación de los padres, etc.), pasando por los factores académicos (opción de bachillerato o ciclo formativo, rendimientos previos...) hasta los determinantes de tipo pedagógico y del papel del alumno en el aprendizaje de la asignatura. En concreto, entre estos últimos, se considera: 1. Variables relativas a la metodología docente basada en la implementación del EEES: Estrategias de evaluación y metodología de la enseñanza. 2. Variables referidas a la dedicación y disponibilidad de tiempo para la asignatura por parte de los alumnos: número de asignaturas en el mismo año-semestre, si trabajan o no, horas de estudio dedicadas a la asignatura, porcentaje de asistencia a clase (Moreno, Ramos y García, 2017, pág. 40).

La anterior cita, cuyo fin es que las directivas y los profesores de la Facultad la tengan en consideración, al momento en que estén diseñando los programas de enseñanza/aprendizaje del inglés, como segunda lengua. En otros términos: es imprescindible que se tengan en cuenta que no son pocos los estudiantes que trabajan, por variados motivos: porque necesitan hacerlo para pagarse sus estudios universitarios, porque debido a su edad era más que normal que ya se encontraran trabajando, al momento de ingresar a la Universidad.

La inteligencia: es preciso tener en cuenta que este es un factor que, cada vez es menos tenido en cuenta, para efectos de analizar la posibilidad de que alguien 
aprenda una segunda lengua, estando inserto en una carrera universitaria. Sobre este controvertido tema, es muy importante tener en consideración algunas teorías de Gardner al respecto, en el sentido de que los estilos de aprendizaje se encuentran relacionados con la idea del aprendizaje como un proceso activo. Teniendo en cuenta que el aprendizaje es la compleja elaboración por parte del receptor de la información recibida, es entonces evidente que el individuo relacionará y procesará la información que recibe en función de sus propias necesidades y no como un simple agente pasivo, como se pensaba hasta hace unos años. En estos llamados estilos de aprendizaje, gran parte de los autores contemporáneos convergen en la teoría de las inteligencias múltiples propuesta por H. Gardner, según la cual cada individuo es dueño de un conjunto de capacidades que le permiten proponer posibles respuestas a las situaciones cotidianas; dependiendo del estímulo que haya recibido, estará en mayor o mejor capacidad de enfrentar el reto que se le proponga, lo que quiere decir a su vez que no todos responderán de la misma (Rincón y Celis, 2017, pág. 78).

De la anterior cita, se colige claramente que la inteligencia, concebida en su exclusiva acepción abstracta, no es la que impera al momento de establecer si la inteligencia misma es determinante cuando de aprender una segunda lengua se trata, sino más bien, de la necesidad de analizar las inteligencias múltiples, que le permiten al individuo responder de diferentes maneras ante los estímulos del entorno.

Actitudes/aptitudes de los profesores: sin un profesorado comprometido y capacitado, es prácticamente imposible diseñar un programa idóneo de enseñanza/aprendizaje del inglés en la Facultad. Aún hay más a este respecto: ante las condiciones específicas actuales del estudiantado de la Facultad, es imprescindible que el diseño de los programas sea realizado por personal altamente calificado. De lo contrario se habrá arado en un desierto porque, si bien es cierto que el trabajo mismo sirve como Tesis Doctoral también es verdad que sólo será útil para ocupar un pequeño espacio en los estantes de la biblioteca de la Universidad de Guayaquil.

Se ha marcado un camino gracias a un trabajo dedicado y realizado con los lineamientos establecidos por el Método Científico. Pero uno de los motivos más relevantes de esta investigación, se constituye por el hecho de que este trabajo investigativo sea de utilidad práctica, efectiva y concreta para la implementación del programa en la Facultad y, posteriormente en toda la Universidad de Guayaquil. Para que ello suceda es preciso que no solo los profesores, sino también, las directivas lo lleven a la práctica estando capacitados para diseñar los programas.

Terminando, entonces, con este acápite de las Teorías Sustantivas consideradas, durante el desarrollo del presente trabajo de investigación, se anota que fueron muchas. Sin embargo, la finalidad de esta Ponencia es plantear lo más importante para referirse a las teorías ya aludidas reforzando su exposición con fuentes bibliográficas más recientes.

\section{Metodología}

Los métodos utilizados para la elaboración de la presente Ponencia, no distaron mucho de los métodos, ni de la metodología misma, para desarrollar el presente trabajo investigativo. Uno de los métodos que hizo parte de la Metodología fue la Validación por Juicio de Expertos, que otorga dos elementos fundamentales, esto es, la Fiabilidad y la Validez del Contenido que se determina, esta última, a partir de dos factores: de un lado, partiendo del diseño de la prueba encuesta $y$, de otra parte, por la validación de dicho instrumento de recogida de datos. Aunque Validez y Fiabilidad 


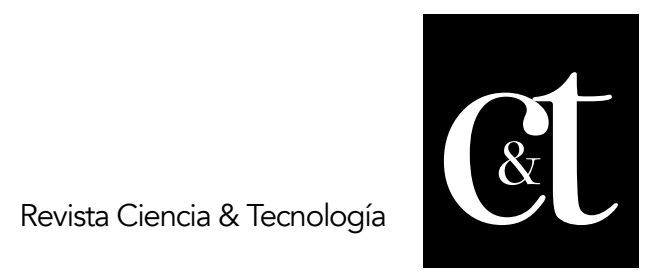

No. 18, 30 de abril de 2018

ISSN impreso: 1390 - 6321

parecen, en principio, "dos caras de la misma moneda", se trata de dos conceptos que tienen sus diferencias conceptuales. La validez brinda fiabilidad. Entonces, cuando un instrumento de recogida de datos es validado por Juicio de Expertos, los resultados que se obtengan luego de la encuesta misma, serán confiables respecto de quienes interesan que lo sean: las directivas y el personal de docentes de la Facultad de Filosofía, Letras y Ciencias de la Educación de la Universidad.

Población y Muestra: en lo que respecta a la población y al tamaño de la muestra para efectos de recoger los datos se recurre a la siguiente ecuación:

$$
n=\frac{N \sigma^{2} Z^{2}}{(N-1) e^{2}+\sigma^{2} Z^{2}}
$$

Cuando se resuelve la ecuación, se obtiene un valor de 309,93 que, si lo expresa en cifras redondas equivale a 310 , que es el número de alumnos que conforma encuestados con las preguntas relativas a la edad, la inteligencia, la motivación y la aptitud/actitud del personal de docentes de la Facultad. Sin embargo, el hecho de que se trata es un muestreo que brinda Fiabilidad a quienes, como las directivas y los profesores, encargados de diseñar los programas de enseñanza del inglés en la Facultad y, por qué no, más tarde, en otras Facultades de la Universidad de Guayaquil. Este es el fin último del presente trabajo de investigación: que los datos recabados sean una guía fiable y válida para quienes diseñarán y, luego, dictarán los cursos.

\section{Resultados}

Tiempo que ha estudiado inglés: 0-5 años: $26 \%, 5-10$ años: $61 \%$, y $10-15$ años: $13 \%$. Este resultado ratifica que la formación en idiomas y, más específicamente, en materia de inglés en el Ecuador es deficiente, como es igualmente deficiente en casi toda América Latina. Sólo el $13 \%$, esto es, prácticamente, una décima parte de los estudiantes universitarios, en general han estudiado el inglés durante la primaria y la secundaria e, incluso, un poco más allá de los estudios secundarios. Ahora, lo más preocupante, se constituye por el hecho de que no se sabe, a ciencia cierta, la calidad 0 , si se prefiere, el nivel de inglés de los alumnos que conforman dicho $13 \%$. De otro lado, tenemos que, si se suma los que han estudiado inglés de 0 a 5 años, más los que lo han hecho de 5 a 10 años, se obtiene los que lo han hecho entre 0 y 10 años, arrojando un total del $87 \%$. En otras palabras, es altísimo el porcentaje de quienes lo han estudiado 10 años o menos. 


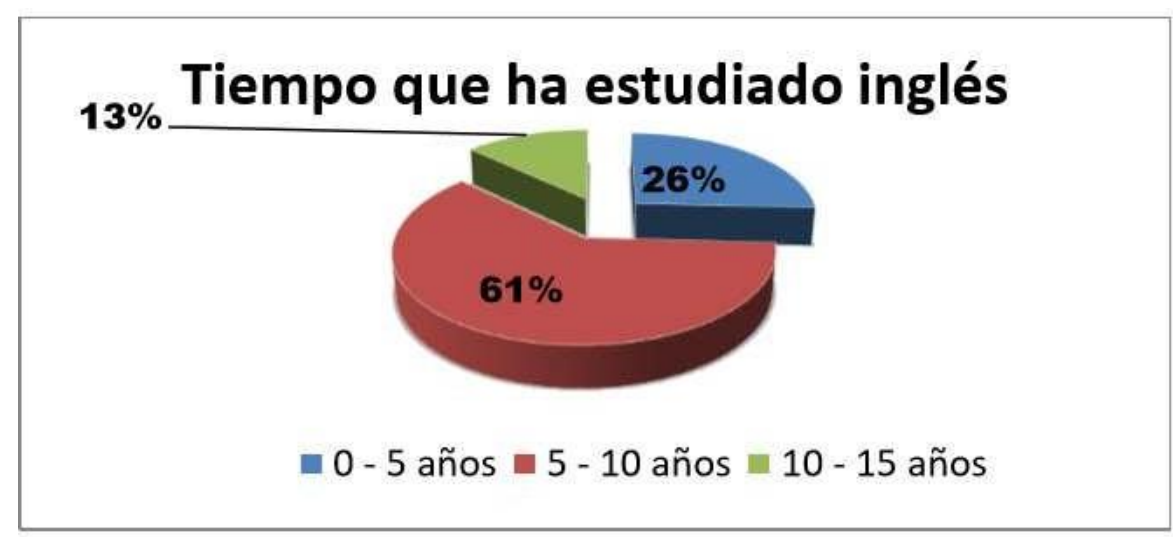

Gráfico 1. Tiempo que ha estudiado inglés

Fuente: Jorge Tapia Celi

¿Cursa usted actualmente alguna materia, curso o clase de inglés, dentro o fuera de la universidad? Sí: $87 \%$ y No: $13 \%$. Este resultado es, paradójica pero afortunadamente, bien alentador, al contrario de lo que sucede con los resultados de la pregunta anterior. $Y$ es alentador, tanto por el hecho de que un altísimo porcentaje de los estudiantes se encuentra estudiando inglés en la actualidad, como por el hecho de que esta situación impactará ostensible y positivamente, en algo que se temía desde un principio: la heterogeneidad en lo que respecta a los conocimientos del inglés, que puede hacer que el proceso se ralentice para quienes están en niveles de inglés más avanzados, provocando problemas para los docentes y, generando algo de desmotivación para los más atrasados. Sin embargo, de lo anterior, no se debe perder de vista que, dentro de ese $87 \%$ hay disparidades, de todas maneras, por cuanto es imposible pretender que todos o que, ni siquiera, una alta porción de dicho grupo, cuenten con niveles parejos porque se trata de estudiantes universitarios y provienen de niveles de educación primaria y secundaria bastante heterogéneos. Además, las disparidades socioeconómicas también determinan ciertas diferencias, porque los más favorecidos son quienes están estudiando inglés en instituciones de más alto nivel.

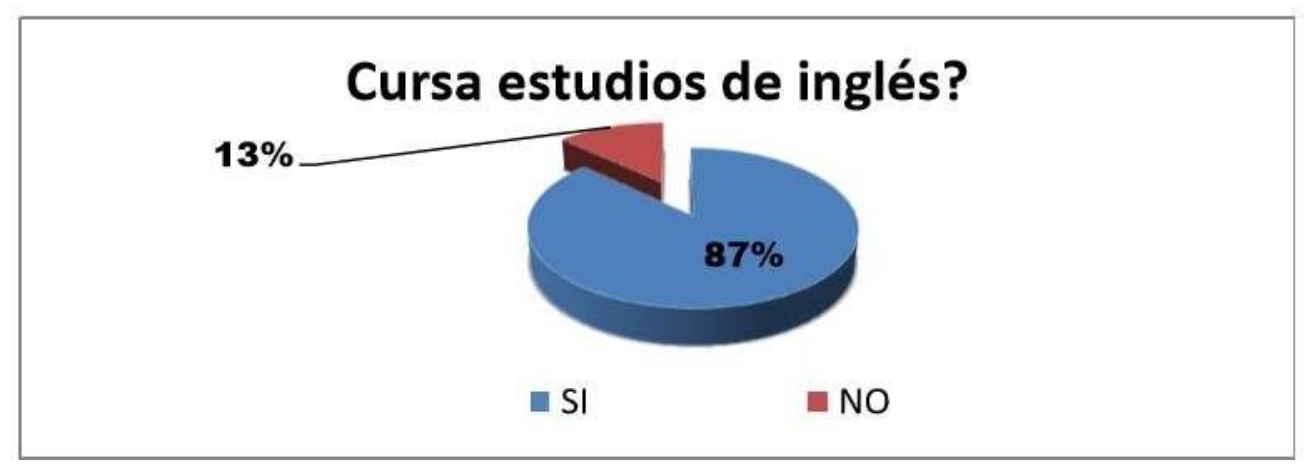

Gráfico 2. Cursa estudio de inglés

Fuente: Jorge Tapia Celi

¿Qué tanto diría usted que domina e idioma inglés? Del total de estudiantes encuestados, el $74 \%$ siente que su dominio es de $25 \%$, el $23 \%$ siente que su dominio 
es de $0 \%$, y el $3 \%$ siente que su dominio es de $75 \%$. Por lo tanto, se puede concluir que los estudiantes tienen dominio mayoritario de 25\%. Análisis: es muy alto el porcentaje de estudiantes de la Facultad que considera que su domino es, tan solo, del $25 \%$. Además, el $23 \%$, esto es, casi la cuarta parte, piensa que su manejo del inglés es nulo.

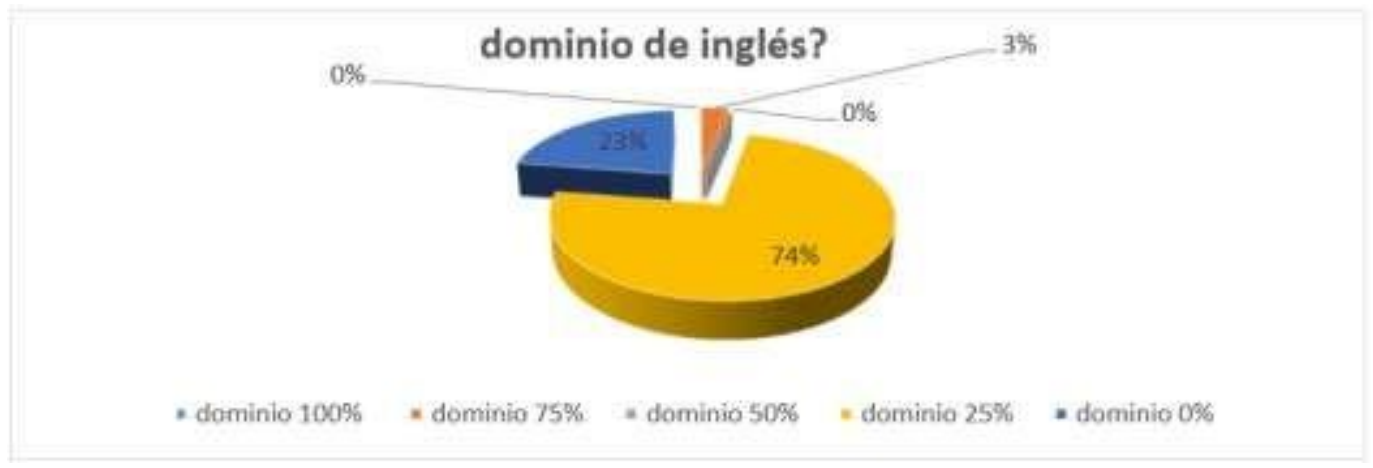

\section{Gráfico 3. Dominio de inglés}

Fuente: Jorge Tapia Celi

¿Siente usted que su edad tiene un impacto sobre su capacidad para aprender y perfeccionar el idioma inglés? No: $61 \%$ y Sí: 39\%. A pesar de este resultado bastante favorable al "No", los profesores y directivas que diseñarán y, luego, ejecutarán los programas de enseñanza de inglés, como segunda lengua, deberán tener en cuenta que la edad sí es un factor muy importante a tener en cuenta, sobre todo, cuando se trata de alumnos de la Facultad que ya pasan de los 35-40 años, edad a partir de la cual, ya comienza a dificultarse el aprendizaje de los idiomas.

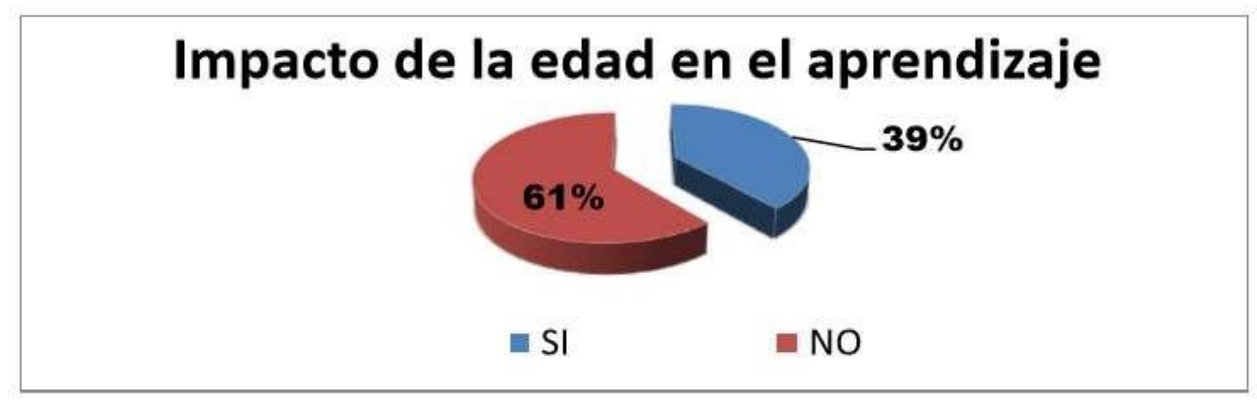

Gráfico 4. Impacto de la Edad en el aprendizaje

Fuente: Jorge Tapia Celi

Si la respuesta es afirmativa ¿Siente usted que su edad afecte su capacidad para aprender y perfeccionar el idioma inglés de manera positiva o negativa? Esta pregunta dio como resultado las siguientes respuestas: Sí: 19\%, No: 49\%, y Ninguna: $32 \%$. Análisis: aunque el resultado global, por así decirlo, es del $81 \%$ (sumando el "No" y el "Ninguna. 


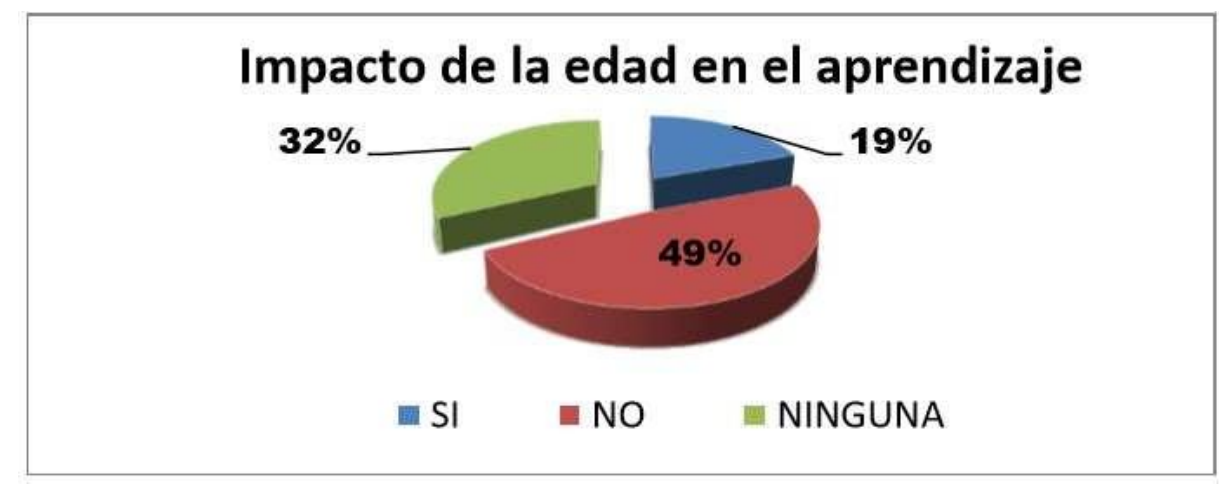

\section{Gráfico 5. Impacto edad}

Fuente: Jorge Tapia Celi

\section{Discusión}

En términos generales, ante resultados sorpresivos, sí se debe tener en cuenta que algunas de estas respuestas no parecen concordar con la realidad científica. Sin embargo, ello no le quita, en lo absoluto, rigor científico a la investigación, ni le resta validez, credibilidad, ni fiabilidad a la metodología empleada, dado que las respuestas que parecen contrarias a la realidad planteada por los expertos, con base en el Método Científico, lo son, por supuesto, pero era absolutamente predecible que, por ejemplo, dijeran que la edad no tiene injerencia en el aprendizaje del inglés, como segunda lengua.

Lo que sucede, realmente, es que la mayoría de los seres humanos, cuando sobrepasa cierto límite de edad, hay la tendencia a desconocer las limitaciones que la edad misma, lleva consigo. Es más, se trata de un fenómeno que, en Psicología, se denomina "Temor a envejecer".

Tratándose, por ejemplo, de Chile, se cuenta que "El estudio Chilescopio, realizado por la consultora Visión Humana a 2.030 personas de las principales ciudades del país, constató que el $49 \%$ de los chilenos reconoce sentir poco agrado ante la idea de llegar a la vejez" (Olivares, 2017, pág. única). Se trata, por supuesto, de un fenómeno generalizado, esto es, de toda la especie humana, el hecho de temer cuando se está envejeciendo, lo que trae consigo la negación de muchos aspectos inherentes al envejecimiento mismo, como es el caso, precisamente, de la dificultad para el aprendizaje, en general, y del aprendizaje de los idiomas, en particular.

\section{Conclusiones}

Plantear una Ponencia sobre un trabajo de investigación extenso y complejo que, además, está elaborado con base en una lista de fuentes bibliográficas, no es para nada sencillo. Fueron muchas las teorías, hipótesis y demás elementos conceptuales tomadas en consideración. Por ende, acá solo se refiere a los que más determinantes fueron a la hora de avanzar en el trabajo.

La revisión de la literatura fue la etapa más compleja de todas, en vista de que son muchas las fuentes que aparecen en el camino, pero no son muchas las escogidas, si se compara su número con el total de fuentes analizadas, así hubiese sido someramente, desde un principio. H. Gardner y, por sobre todas las cosas, algunos de los apreciados profesores, fueron de enorme ayuda para salir de semejante 
"encrucijada" que significa encontrarse, de pronto, con decenas de obras.

En cuanto a los Resultados, se manifiesta que no fueron sorpresivos, también es verdad que se encuentran algunas discordancias frente a las posturas científicas aludidas durante la investigación. Tal es el caso, del hecho de que la mayoría de los alumnos encuestados considera que la edad no es determinante, al momento de estudiar un idioma extranjero cuando, en realidad, sí lo es y está demostrado con creces.

\section{Referencias bibliográficas}

Moreno, B., Ramos, C. y García, A.S. (2017). Efectos de las Estrategias de Enseñanza y Aprendizaje Universitarias en el Rendimiento Académico. Revista d'Innovació Docent Universitària Núm. 9 (2017), pp. 39-53 Visto el 06 Ene 2017 en la URL: <http://revistes.ub.edu/index.php/RIDU/article/viewFile/RIDU2017.9.4/20 $378>$

Olivares, I. (2017). La mitad de los Chilenos Teme Envejecer. Web. Visto el 06, Ene, 2017, en la URL: http://www.latercera.com/noticia/la-mitad-los-chilenos-temeenvejecer

Paíno T. (2017). Evaluación de la Inteligencia Lingüística en la Educación Infantil según el Modelo de Gardner. Universidad de Valladolid. Visto el 06 Ene 2017, en la URL: <http://uvadoc.uva.es/bitstream/10324/26853/1/TFG-G\%202588.pdf>

Rincón, J. y Celis, M.C. (2017). Estilos de Aprendizaje Aplicados a una Segunda Lengua. Colección Académica de Ciencias Sociales. Colección Académica de Ciencias Sociales, 4(2): 75-81. Visto el 06 Ene 2017, en la URL: $<$ https://revistas.upb.edu.co/index.php/cienciassociales/article/download/7535 16864

Robles, P. y Rojas, M. (2015). La validación por juicio de expertos: dos investigaciones cualitativas en Lingüística aplicada. Revista Nebrija de Lingüística Aplicada, 18. Visto el 06, Ene, 2017, en la URL: <http://www.nebrija.com/revista-linguistica/la-validacion-por-juicio-deexpertos-dos- investigaciones-cualitativas-en-linguistica-aplicada> 